\title{
Education Research on the Thinking Method of College Physics Using the Way of Knowledge Mining, Inducing and Inspiring
}

\author{
Chun-qing $\mathrm{Xu}, \mathrm{Li}$ Yang \\ School of physics University of electronic and science technology of China, Sichuan Province, China
}

\begin{abstract}
It is very important to teach thinking methods in the college physics course. Aiming at the phenomena that the knowledge is taken care of and the thinking method is ignored in college physics teaching, we use the way of knowledge mining, inducing and inspiring to discuss how to teach thinking methods of college physics in the limited class hour, and how to make students digest and absorb these thinking methods in order to cultivate students' scientific quality, improve their ability of knowledge learning and problem solving. The teaching effects of this method are analyzed by way of questionnaire.
\end{abstract}

Keywords - college physics, thinking, method

\section{挖掘式、归纳式和启发式大学物理思想方法教学探索}

\author{
许春青＼cjkstart杨莉 \\ 电子科技大学物理电子学院, 成都, 四川, 中国
}

摘 要 在大学物理教学中思想方法的讲授是非常重要的, 针对当前大学物理教学环节中普遍存在重知识、轻思想方法的现象, 探讨了通过知识挖掘, 归纳和启发的方式在大学物理有限的课时中如何将物理思想方法融入到教学中、如何使学生消化和吸收这些思 想方法, 以达到提高他们的科学素养、培养学习和研究问题能力的目的。并采用问卷调查的方式对大学物理思想方法课堂讲授效果进 行了分析。

关键词 大学物理, 思想, 方法

\section{1. 引言}

与大学英语和大学数学等其它课程不同, 大学物理中 包含了几百年来物理学先辈们分析问题和解决问题的一系 列思想和方法。授人以鱼, 不如授人以渔, 在大学物理教 学中思想方法的讲授是特别重要的, 在素质教育的大环境 下, 这一问题得到了越来越多人的重视[1]。但几乎还没有 那一本大学物理教材对物理思想方法给予较为系统的阐 述, 也很少有大学物理教师在授课过程中对这部分内容给 予重视。物理思想和方法蕴涵在知识当中, 在学习过程中 绝大多数学生在掌握知识的同时很难将它们挖掘出来, 进 而对其有所领悟。这就需要大学物理教师们通过挖掘和归 纳的方式将这些宝贵的物理思想和方法融入到课堂教学 中, 并通过启发的方式使学生们能够理解、领悟和运用好 这些思想和方法, 从而提升他们的科学素养、独立思考能
力和研究问题能力。

学术界对于大学物理思想和方法的课堂讲授做了不少 探索, 朱鈸雄提出了大学物理思想方法资源库建设的三部 曲[2], 即分别由《物理学方法概论》、《物理学思想概论》 和《大学物理学习导引一一导读、导思、导解》三部课外 教材作为正式教材的补充。郑春红从理想模型、分析综合、 归纳演绎和类比法等四个方面阐述了如何在大学物理课堂 中讲授思想方法的问题[3], 此外还有侯新杰[4]、刘海兰等 人的研究 [5]。在这些研究中, 有的缺乏相应的案例, 有的 可操作性不强。本文通过挖掘、归纳和启发的方式对在大 学物理有限的课时中如何将物理思想和方法融入到教学中 和如何使学生消化并吸收这些思想方法做了有益的探索, 通过知识的挖掘和归纳拓展学生的知识体系, 使这些思想 和方法融入到大学物理的教学中, 增强学生的认知能力, 
通过丰富的课堂案例提高学生对这些思想方法的理解和领 悟, 通过不断的启发强化学生对这些思想方法的消化和吸 收。我们的做法在一定程度上弥补了当前大学物理教学中 普遍存在的重知识、轻思想方法的弊病。

\section{2.学习物理思想, 提高科学素养}

物理学研究问题的思想涉及方方面面, 结和大学物理 的学习, 我们主要从四个方面概括这部分内容, 分别是理 论的来源、理论的检验、理论的发展和理论的评价。在讲 到大学物理中的定律时, 例如牛顿定律和能量按自由度均 分定律等, 作为知识体系的拓展, 从什么是定律和什么是 定理引申出这部分内容的讲授。这些基本思想就犹如物理 学大路上的“交通规则”, 具备这些基本思想不仅可以加深 物理知识的理解, 使研究问题的思路更清晰, 还能避免碰 壁和少走弯路, 是一个研究者必须具备的基本科学素养。

物理学是由定律 (或原理) 及定理所组成的一门逻辑 严密、具有系统性的科学。物理学中的定律来源于实践, 是由实践总结而来的, 而定理是由定律经过严密的逻辑推 导而得到的, 所以物理学的最终来源是实践。物理学的理 论体系好比一棵大树, 定律 (或原理) 就是它的根, 由定 律推导而来的定理就如同它的干和枝, 而人们的实践活动 则是其生存的土壤, 区分不清楚哪些是理论的根, 哪些是 它的干和枝, 自然就很难把握好所学的知识, 也就很难应 用好它。例如, 为什么一个物体加速度的大小与合外力成 正比, 与物体的质量成反比? 为什么变化的磁场能在空间 激发浴旋电场? 等等这类问题是许多人经常犯的一个错 误, 即对物理定律 (或原理) 问为什么, 这是对理论来源 认识不清的一个典型例子, 定律是由实践总结而来的, 不 是推导而来的, 所以没有“为什么”。

由于定理是由定律推导而来, 其正确与否要看推导过程 以及推导所依据的定律是否正确, 那么定律正确与否的判 断依据是什么呢? 答案是实践。理论来源于实践, 其正确 与否必须受到实践的不断检验, 这是检验理论的唯一标准。 例如, 人们长久以来都坚信的一些原理 (或定律) 以及学 术权威等都是绝对靠得住的么? 答案是否定的, 因为实践 才是检验理论的唯一标准。这是一个创新型人才所必须具 备的基本科学素养, 创新就要放开手脚, 倘若敬畏传统、 敬畏权威, 难免被束缚住思想。

随着物质技术手段的不断提高, 人类的实践活动也会 逐步深入。这就导致物理定律在一定时期内与人们的实践 相符, 但随着实践活动的深入, 与这些定律不相符的现象 就可能会出现。人们就需要提出新的假说, 新的假说经得
起实践的检验就成为新的定律, 这就导致理论的发展过程 是一个渐进的过程。正如波普尔的观点: “任何理论都不是 绝对正确的永恒真理, 而只是暂时的假设”。新理论必须与 原有理论之间存在衔接, 即新理论会指出原有理论适用于 某一范围内, 例如狭义相对论指出牛顿力学适用于低速情 形, 如果没有这一衔接, 原有理论所能解释的事实新理论 则可能解释不了, 从而产生理论与实践的冲突。例如, 如 果将来物质运动超光速的现象或者磁单极子被发现, 我们 今天所学的狭义相对论和电磁学就会被抛弃么? 答案是否 定的, 如果这些与我们所学理论相冲突的现象被发现, 则 是未来新理论诞生的实验基础, 未来的新理论会指出我们 今天所学的狭义相对论和电磁学是某一范围内的近似理 论, 在该范围内狭义相对论和当今的电磁学理论依然适用。

理论的正确性由实践来检验, 但评价理论就不仅如此 了。不同领域理论的评价标准也有所不同, 但有实践基础、 应用范围广泛、逻辑简单、应用方便是评价一个理论好坏 的基本标准。正如人际交往需要有底线和标准一样, 学习 和理论研究同样离不开理论好坏的判断标准, 否则, 好坏 不分, 怎么可能理解并应用好所学的知识。例如, 一个很 深奥、所用数学很复杂的理论就是很了不起的理论么? 答 案是否定的, 我们评价一个理论的好坏, 不是看这个理论 是否应用了多么复杂的数学工具, 更不是看这个理论是否 高深莫测, 那些逻辑简单并且解决问题方便的理论才是值 得我们推崇的。

\section{3.学习物理方法, 培养学习和研究问题能力}

物理方法有很多, 本文以物理学最常见、最基本的理 想模型为例, 来分析如何结合大学物理的具体内容来讲述 这些研究方法, 如何启发学生, 以提高研究问题能力; 以 理论的基本结构框架为例, 来分析如何结合大学物理的具 体内容, 使学生建立起知识的结构框架, 培养学生将知识 系统化的能力, 进而提高学习能力。

\section{1 分析问题和解决问题能力的培养——理想模型}

质点、刚体和理想气体等都是典型的理想模型, 在讲 授这些内容时, 可以作为知识体系的拓展, 把这一物理学 最基本的研究问题方法讲授清楚。我们从什么是理想模型、 为什么要应用它、为什么它重要、它的核心思想是什么、 如何看待理想模型所造成的偏差、如何建立理想模型以及 如何改进理想模型等几个方面来叙述。这也是物理学最重 要的研究问题方法之一, 每一个学过大学物理的学生都应 该掌握好它。所谓理想模型就是抓住问题的主要因素, 舍 
弃某些非本质的次要因素, 把实际问题理想化。现实问题 是非常复杂的, 事无巨细地都加以考虑往往使问题难以得 到解决, 正因为如此, 理想模型这种研究问题方法的应用 是极为广泛的。理想模型所体现出的思想就是 “近似地处理 问题”, 从一般意义上讲, 凡是近似地处理问题的做法都可 以称之为理想模型。既然理想模型是现实情形的近似, 那 么利用它来分析问题, 其结果必定与实际有偏差, 我们引 入理想模型的意义就在于如果这个偏差比较小, 满足我们 的精度要求, 该模型就不失为一个成功的、有应用价值的 模型; 倘若该偏差比较大, 超出了我们的精度要求, 则需 要对这个模型加以修正, 一般的做法是将忽略掉的某些比 较重要的因素重新考虑进来。对于一个理性模型来说, 往 往存在一个应用的范围问题, 超出了这个范围, 所带来的 偏差就可能很大, 该模型就需要进一步修正。例如理想气 体在研究压强和温度时比较成功, 但在研究能量问题时就 产生了不能接受的偏差, 就需要修正, 把气体分子看成自 由、弹性的质点组。建立理想模型需要对所研究的问题有 深入细微的了解, 只有这样才能分清主次因素, 从而把实 际问题简化。

在物理学中, 理想模型的例子比比皆是, 除了前文提 到的质点、刚体和理想气体之外, 电介质分子正负电荷中 心的概念是一个非常典型的理想模型。显然电介质分子中 所有正电荷产生的电场不可能严格等效于一个集中了所有 正电荷电量的点电荷所产生的电场, 负电荷产生的电场也 一样, 但做这样一个大胆近似后, 一个电介质分子放入电 场中所发生的变化就很容易分析了, 进而在电场中电介质 表面出现的极化电荷也就容易理解了。如果不做这样的近 似处理, 那么电介质在电场中所发生的变化分析起来就很 复杂。在康普顿散射一节中, 将光子和外层电子碰撞看作 是和自由电子碰撞, 将光子和内层电子碰撞看作是和整个 原子碰撞, 这也是一个非常典型的例子。我们讲到这些例 子时都会将相关知识加以挖掘, 并启发学生, 以后遇到类 似问题能不能像物理前辈这样大胆近似处理, 从而解决实 际问题。理想模型给我们很多启示, 它不仅是一个基本方 法, 也是分析问题的一个基本思路, 在面临实际问题时首 先应该想到去分析哪些是主要因素, 哪些是次要因素, 然 后通过忽略次要因素的方式将实际问题简化并解决。不仅 如此, 在学习过程中也可以对前人的理论有很好的理解。

\section{2 系统化知识能力的培养——理论的基本结构框架}

不同种类动物之间差别巨大, 但一般都有类似的结构, 不同理论体系之间差别也很大, 但一般也都有类似的结构
框架。即一个理论体系往往可以分为运动学和动力学两个 部分, 这两部分内容就组成了一个理论体系的基本结构框 架。我们就运动学包括哪些内容、动力学包括哪些内容、 这个结构框架的应用和启示等几个方面分别叙述。在课堂 讲授中, 从质点运动学、理想气体状态参量等处, 作为知 识体系的拓展, 引申出这部分内容的讲授。

一个理论体系的运动学一般涵盖引入物理量来描述研 究对象并给出这些量之间的关系, 而动力学则一般包含从 实践中总结出定律, 给出这些物理量是如何变化的。比如 在质点运动学中引入位置、速度、加速度、能量、动量和 角动量等物理量来描述质点的运动, 并给出了这些量之间 的关系; 在质点动力学中牛顿定律给出这些物理量是如何 变化的, 对牛顿第二定律所得到的加速度积分就可得到速 度和位置如何随时间变化的, 进而就可以得到能量、动量 和角动量如何随时间变化的。

在学习一门理论时可以用这一物理学基本结构框架将 所学知识系统化, 清楚了该理论引入了什么变量来描述研 究对象、这些量之间的关系是什么以及能给出这些变量如 何变化的定律是哪个, 所学理论体系的轮廓就清晰了。该 理论结构框架的另一个重要启示是在做研究时也可以效仿 它, 即引入变量来描述研究对象, 之后由实践总结出定律, 给出这些变量是如何变化的, 进而将所要研究的对象描述 清楚, 一旦自己这样的做法获得成功, 往往是重大的理论 贡献。

我们仅以量子力学为例, 分析运用该结构框架如何将 其系统化。在量子力学中几乎没有“量子运动学”和“量子动 力学”这样的提法, 但在量子力学中有运动学和动力学这两 部分的内容。波函数、力学量的算符表示和状态叠加原理 属于量子力学的运动学部分, 这部分内容给出了量子力学 研究问题所引入的物理量及它们之间的关系。薛定谔方程 则属于量子力学的动力学部分, 薛定谔方程给出了波函数 如何随时间变化的, 由于各力学量的平均值是由相应的力 学量算符作用在波函数上得到, 清楚了波函数如何变化的, 各力学量如何变化的也就清楚了。

学习应该善于“一以贯之”, 这样所学的知识才形成体 系。该物理学基本结构框架就是系统化所学知识的一个重 要方式, 不仅能拓展学生的视野, 而且在这一过程中所进 行的思考也非常有利于知识的消化和吸收。这一物理学基 本结构框架就如同樵夫砍柴的绳子, 在学习过程中用它将 砍的“柴”捆好。

对于其它物理思想方法的挖掘, 归纳和启发, 本文无法 一一尽述, 对于我们在课程中讲授的这部分内容, 见表 1 。 


\section{4.物理思想方法课堂讲授的效果分析}

本文用问卷调查的方式来考察物理思想和方法的讲授 效果, 在讲授之前和之后分别在班上做问卷调查, 通过对 比两次调查结果来考察教学效果。

在讲授物理思想和方法之前的问卷调查表明, 在 249 份有效问卷中有 $55.02 \%$ 的同学认为非常有必要在大学物 理课堂中讲授物理思想和方法, 认为有必要的同学占 36.95 , 认为无所谓和没必要的同学占 $8.03 \%$ 。这一调查结 果和文献[6]的基本一致, 表明学生们普遍认为物理思想和 方法是非常重要的, 并对课堂中讲授这部分内容有较高的 认同和期望。
在讲授物理思想方法之后的问卷调查表明, 在 236 份 有效问卷中 $74.85 \%$ 的同学认为笔者在课堂上所讲授的物 理思想方法非常有意义。有 $47.9 \%$ 的同学详尽地表述了这 一看法, 比如很有启发, 收获很大, 对解决类似问题很有 帮助, 让自己开始学会独立思考, 使自己在思想上获得很 大提高, 像一酉灯指引自己的学习和研究, 使所学知识更 加清晰、有序, 对知识有个更系统的了解等等。由两次问 卷调查结果可以看出, 笔者对于物理思想方法的讲授达到 了学生们的预期, 在培养思维能力、提升科学素养和增强 研究问题能力方面效果显著。

表 1： 其它物理学研究问题的思想和方法

\begin{tabular}{|l|l|}
\hline 思想和方法 & 内容简介 \\
\hline 分析与综合法 & 将复杂事物分解为各简单的部分分别加以研究, 将事物各个部分按内在联系统一为整体 \\
\hline 归纳和演绎法 & 分析众多事例并概括出其规律, 由一般到个别的推理方法 \\
\hline 特殊情景法 & 一个难以解决的复杂问题, 找出它容易解决的特殊或极端情况来研究, 也能得到有意义的结论 \\
\hline 类比法 & 与原有理论做类比 \\
\hline 量体裁衣法 & 比照实验结果提出新理论 \\
\hline 逆向思维法 & 比照现有理论, 逆向思考问题, 可以为研究问题提供了一个方向 \\
\hline 在继承的基础上创新 & 研究新问题时, 从原有理论中寻找可以借鉴的地方 \\
\hline 解决问题要有灵活性 & 一个小的变通可以带来很多便利, 解决很多问题, 甚至让不可能成为可能 \\
\hline 要抓住研究对象的特性 & 利用研究对象的特性提出新的概念和理论 \\
\hline
\end{tabular}

\section{5.结束语}

授人以鱼, 不如授人以渔, 在越来越重视素质教育的 今天, 大学物理教学中只重视知识、不重视思想方法的现 象需要加以改变。我们通过挖掘、归纳和启发的方式将物 理思想方法穿插于各教学章节中, 在培养思维能力、提高 科学素养和增强研究问题能力方面收到了比较满意的效 果。特别是那些致力于将来从事科研工作的同学在听了笔 者的大学物理课程后表现出了很高的认同, 都能基本掌握 上述物理学基本思想、基本方法、基本结构框架, 他们所 学习的知识有了一定的系统性, 对物理问题的分析和研究 有了较为清晰的思路, 这在他们学习和将来工作中的作用 是不言而喻的。我们的这些探索在一定程度上弥补了当前 大学物理教学环节中的一些不足。

\section{参考文献(References)}

[1] J. F. Zhao, K. L. Huang, "Research on innovation of college physics teaching content and method," High Science Education, no.6, pp.73-77, June 2002.
[2] H. X. Zhu, X. H. Wang, "The Construction and executive policy of education resource of college physics thinking method," Physics and Engineering, vol. 22, no. 3, pp. 41-47, March 2012.

[3] C. H. Zheng, F. F. Wang, "Strengthen the training of scientific thinking methods, improve the teaching level of college physics," Physics and Engineering, vol.22, no.3, pp. 52-60, March 2012.

[4] X. J. Hou, Y. Zhang, "Permeate physical method education, improve the students' scientific quality," Modern Physics Knowledge, no. 1, pp. 55-57, October 1998

[5] L. Hai, "Pay attention to physics thinking method teaching," Physics and Engineering, vol. 19, no. 6, pp. 17-26, June 2009

[6] H. Q. Wu, "Analysis on educational goal and teaching strategies of scientific methods," Journal of the Chinese Society of Education, no. 10, pp. 46-49, October 2010. 